\title{
MODELO DE BASE DE DATOS PARA LA ELABORACIÓN DE HERBARIOS VIRTUALES
}

\section{DATABASE MODEL FOR THE ELABORATION OF VIRTUAL HERBARIUM}

\author{
María José Velasco Jiménez; Juan Manuel Delgado Marzo; \\ Alberto Álvarez de Sotomayor Posadillo; Francisco Villamandos de la Torre; \\ Carmen Galán Soldevilla* \\ * bv1gasoc@uco.es
}

Universidad de Córdoba

Received: 30/06/2017_ Accepted: 25/06/2018

\begin{abstract}
An adaptive database, with an application throughout the professional life of the students, has been developed in this project, with didactic purposes to help identify the vegetative characteristics of common plants in the Sierra de Córdoba. The purpose is to include this tool in the materials of the subjects taught in the Department of Botany, Ecology and Plant Physiology. This database uses mainly the leaf as character for the identification of species, also including information about the stem, inflorescence, flower or fruit. It has been tried to construct an adaptive instrument of aid in the development of the expert domain in the identification of vegetal species that every biologist must possess for its professional performance.
\end{abstract}

Keywords: Data base; virtual herbal; leaves

\section{Resumen}

En este proyecto se ha elaborado una base de datos adaptativa, y con aplicación a lo largo de la vida profesional del alumnado, con fines didácticos para ayudar a la identificación de características vegetativas de plantas comunes en la Sierra de Córdoba. El objeto es incluir esta herramienta en los materiales de las asignaturas que se imparten en el Departamento de Botánica, Ecología y Fisiología Vegetal. Esta base de datos utiliza principalmente la hoja como carácter para la identificación de especies, incluyendo también información sobre el tallo, inflorescencia, flor o fruto. Se ha pretendido construir un instrumento adaptativo de ayuda en el desarrollo del dominio experto en la identificación de especies vegetales que todo biólogo debe poseer para su desempeño profesional.

Palabras clave: Base de datos; herbario virtual; hojas

\section{INTRODUCCIÓN}

La implantación del Espacio Europeo de Educación Superior está llevando al profesorado a la búsqueda de nuevos métodos de formación. Este nuevo modelo ha propiciado un aumento en el número de horas prácticas que se imparten. Las clases prácticas son un buen momento para implicar al alumnado en el proceso enseñanza-aprendizaje ya que se suele emplear en ellas una metodología más activa que en las clases teóricas.

La identificación de material biológico, gracias al desarrollo de la capacidad de reconocimiento de los diferentes organismos, y en concreto organismos vegetales, es una competencia a desarrollar en distintos grados, como ejemplo en Biología y Ciencias Ambientales que se imparten en la Facultad de Ciencias de la UCO.

Para ello es necesario desarrollar competencias como el saber plantear y resolver problemas asociados a las ciencias en la vida cotidiana, desarrollo de procedimientos propios de la Botánica, adquisición de destrezas prácticas en la metodología de la clasificación propia de la disciplina y capacidad de reconocimiento de organismos vegetales para poder tener una visión actualizada del mundo vegetal que nos rodea.

En definitiva, los profesionales en formación que se inician en la identificación de organismos biológicos deben ir construyendo poco a poco su propio "ámbito experto de reconocimiento de especies" (vegetales en este caso) que van a ir utilizando en ulteriores trabajos a lo largo de todo su desempeño profesional. Este es, por tanto, un trabajo que se considera clave y que se desarrolla durante un tiempo muy prolongado (normalmente se dilata a lo largo de muchos años), necesitando una verdadera implicación del aprendiz en el desarrollo de aquello que hemos denominado "ámbito experto".

Como docentes, hemos pretendido enfrentarnos al reto de ayudar en este proceso de construcción de conocimiento experto de nuestro alumnado aportando un instrumento versátil, potente y adaptativo, que le permita ir construyendo y mejorando ese ámbito experto, no solo en el desarrollo de la asignatura, sino también a lo largo de su futuro profesional.

La idea es que el alumnado no sea un mero observador de las características de los organismos vegetales en campo o en el laboratorio, siendo el profesorado el que le facilita el nombre de la especie de que se trata, sino que sea un proceso más autónomo en el que los estudiantes trabajen los conceptos vistos en clase y les sirvan para llegar a solucionar el problema al que se enfrentan, gracias a la ayuda de esta base de datos. En este 
sentido, esta innovación se ha concebido como una herramienta que facilita un aprendizaje experiencial en el sentido inicialmente propuesto por Dewey (1997), y desarrollado posteriormente por autores como Kolb (2001). Para Dewey, el aprendizaje experiencial es activo y utiliza los ambientes físicos y sociales para extraer lo que contribuya a experiencias valiosas, tratando de establecer un fuerte vínculo entre el aula y el entorno.

\section{OBJETIVOS}

El objetivo general de esta innovación ha sido dotar al alumnado de una herramienta de trabajo que promueve el aprendizaje experiencial, de manera que pueda aprender a identificar de forma autónoma, y en contacto con su medio natural, algunos de los organismos vegetales presentes en nuestra sierra.

Asimismo, como objetivos específicos, nos hemos propuesto mejorar en el alumnado el desarrollo de las siguientes competencias:

- Capacidad de integrar las evidencias experimentales encontradas en los estudios de campo y/o laboratorio con los conocimientos teóricos (CB5).

- Aprender el manejo de las bases de datos y de programas informáticos que pueden emplearse en el ámbito de las Ciencias de la Vida (CE6).

- Capacidad de interpretar la biodiversidad del medio natural, la estructura, fisiología y funciones de los seres vivos y los conceptos de evolución, taxonomía y desarrollo (CE11).

- Capacidad de identificar especies animales y vegetales como parte de los recursos naturales de nuestro país, su adaptación y conservación (CE13).

- Manejar los procedimientos más frecuentes para macrofotografía en campo (CE14).

\section{Metodología}

Los participantes del proyecto han realizado previamente varias salidas a diferentes zonas de la Sierra de Córdoba para seleccionar las especies más significativas a incluir en la base de datos. Se ha tratado de incluir especies que estén también presentes en espacios naturales para que sean fáciles de encontrar y se puedan utilizar también en las salidas al campo.

Una vez seleccionadas las especies se ha procedido a fotografiar y estudiar las características de la hoja de dichas especies, como carácter principal para la identificación de cada una.

Para la elaboración de la base de datos se ha utilizado el programa Microsoft Access del paquete Office 2016. Esta base de datos se ha iniciado por el profesorado, a modo de ejemplo significativo, con un número de especies seleccionadas. A partir de este trabajo inicial será cada alumno, de forma personal, quien vaya construyendo su propia base de datos de especies vegetales que él mismo sea capaz de reconocer. En primer lugar, modificando y sustituyendo las fotografías aportadas por el profesorado para cada una de las características de las especies seleccionadas; en segundo lugar, añadiendo nuevas especies, sus características para la identificación y las fotografías ilustrativas de las mismas.

\section{DESCRIPCIÓN}

La herramienta que hemos puesto en marcha es el germen de una base de datos personalizada para cada estudiante. El modelo de base de datos que se ha realizado incluye campos descriptivos de las características vegetativas de una serie de especies (árboles y arbustos, principalmente) que van ligados necesariamente a una fotografía en la que se reconoce y destaca una de esas características. Se han escogido sólo aquellas plantas que están presentes en el área elegida para el desarrollo de las prácticas de campo.

Es importante hacer notar que, en la definición de cada uno de los registros a incluir, el alumnado deberá demostrar de una forma muy evidente (para el profesorado y el proceso de evaluación) que sus anotaciones de características para el reconocimiento son verdaderamente relevantes para él. Esto se pretende conseguir solicitando para cada una de las características seleccionadas una fotografía que ejemplifique de forma nítida lo que se describe.

La base de datos ha utilizado las características de la hoja como objetivo principal para la identificación de especies. No obstante, se ha incluido información complementaria sobre las características del tallo, inflorescencia, flor o fruto, cuando éstas han resultado relevantes en el proceso de identificación.

La base de datos consta de una serie de tablas relacionadas entre sí. Existe una tabla principal (FOT_BOT) y otra de información subordinada (EVALUA_F). Estas dos tablas principales se relacionan entre sí a partir de un campo principal (Id). Con ellas se relacionan, directa o indirectamente el resto, que funcionan como tablas diccionario (Figura 1). 


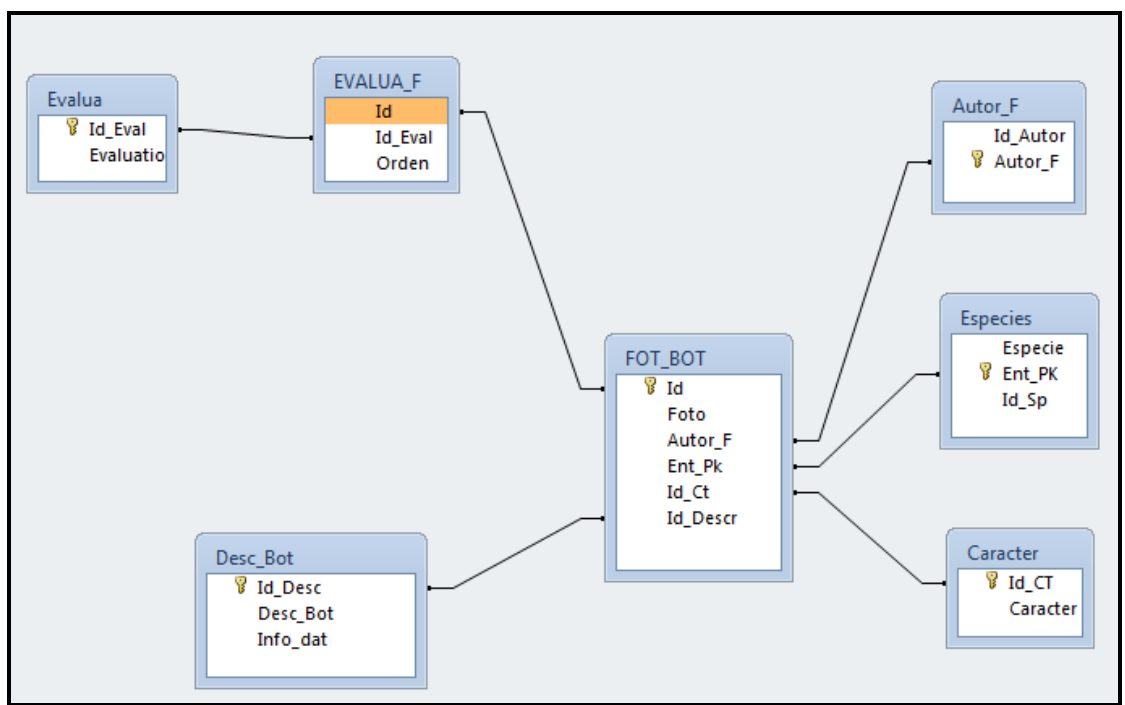

Figura 1. Diseño de las relaciones de las tablas que conforman la base de datos.

En la tabla principal, además de la mencionada clave principal (Id), se localizan los datos descriptivos de la imagen que debe aportar el estudiante. En primer lugar, el campo Foto, incluye el hipervínculo con el fichero de imagen al que se refiere cada registro.

El campo Autor_F se ha incluido al objeto de que se indique la autoría de cada imagen, que podría no corresponderse con la persona que deba generar la base de datos, aunque se entiende que esto debe ser una excepción. De esta forma, en todo caso, se respeta la autoría real de las imágenes que se incluyen en la aplicación.

Los otros tres campos se abastecen de las correspondientes tablas diccionario donde se incluyen las posibilidades entre las que deberá elegir el alumnado a la hora de cumplimentar la base de datos.

La decisión de usar campos codificados, en lugar de incluir datos de texto directamente, se ha tomado al objeto de prevenir la necesidad de modificar registros ante cambios nomenclaturales (relativamente frecuentes en taxonomía) o bien atender a las variaciones de criterio a la hora de redactar los contenidos que pudieran surgir por parte del profesorado usuario.

Sin embargo, aunque se han diseñado como campos numéricos, estos se traducen de forma automática a través de las tabas diccionario, por lo que en apariencia se interpretarían como campos de texto. Para ello, se ha diseñado un formulario de inclusión de datos. Aunque el diseño del formulario no es un aspecto fundamental para el resultado final, sí se considera una herramienta de utilidad para que la recopilación de datos se realice de forma más lógica y fácil.

\section{RESULTADOS OBTENIDOS}

El resultado del proyecto no es otro que el propio modelo de base de datos en sí, concebido como una herramienta útil para facilitar un proceso de enseñanza-aprendizaje significativo. Dado que el modelo de datos se manifiesta a través del formulario que se ha diseñado, se procederá a la descripción del mismo.

El formulario diseñado para facilitar la inclusión de los datos se ha denominado "FOTOS", a partir del que se puede dar cumplimiento a la totalidad de las tablas implicadas en el diseño de captura de información previsto. En el formulario, los campos aparecen "traducidos" de forma que sea más intuitiva la interpretación del mismo. Así, por ejemplo, los campos Ent_pk, Id_Ct e Id_Desc se describen como "Especie", "Carácter" y "Descripción", respectivamente (Figura 2). 


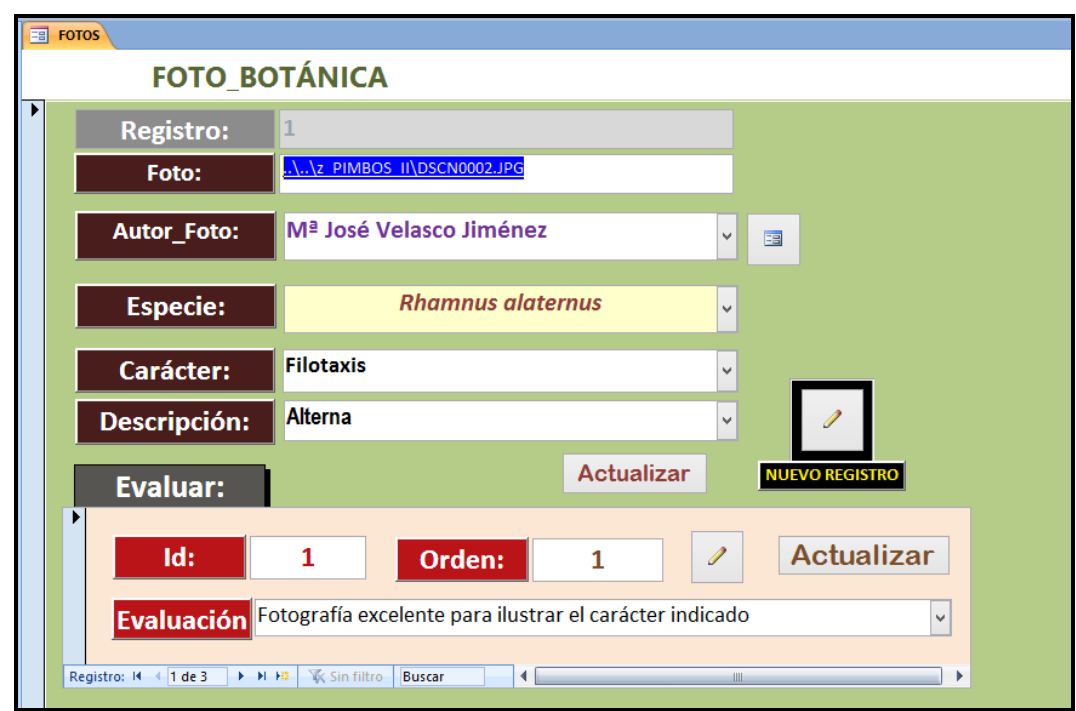

Figura 2. Vista del formulario "FOTOS", con un ejemplo cumplimentado.

El campo Ent_pk, (Especie) se cumplimenta activando una pestaña desplegable en la que aparece la relación de especies ordenadas alfabéticamente, entre las que debe elegir el alumnado, reduciendo los problemas derivados de los errores de tecleo (Figura 3).

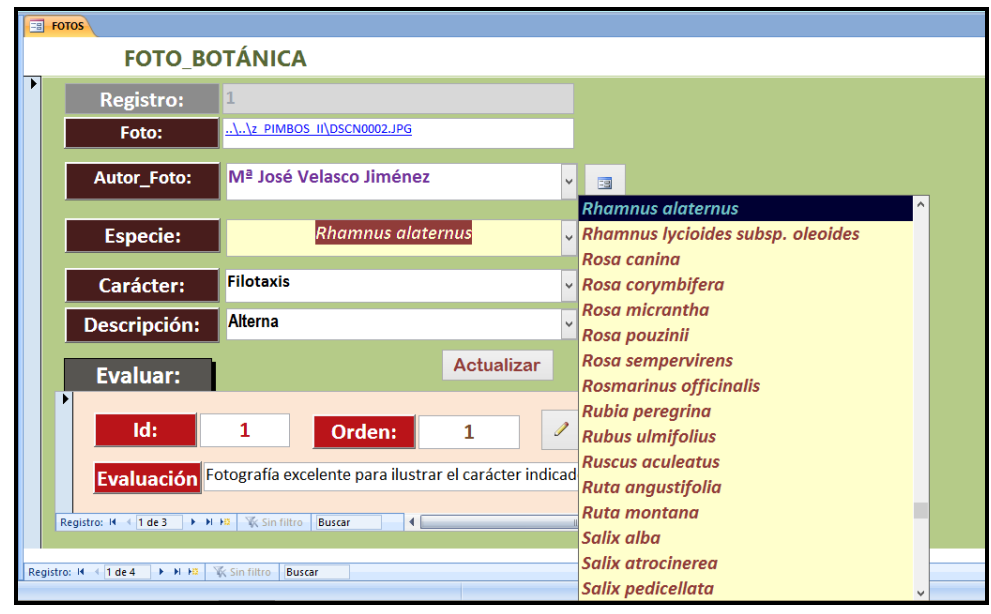

Figura 3. Recreación de la pestaña desplegable para la selección de la especie con la que se corresponde la fotografía.

El desplegable se refiere a la tabla ESPECIES, donde el profesorado tiene la posibilidad de incluir las especies de plantas que considere oportunas. En principio, se han ofrecido las 40 especies de plantas arbóreas y arbustivas más frecuentes en Sierra Morena, en el entorno de la ciudad de Córdoba, ya que esta es el área en la que se llevan a cabo las clases prácticas de las asignaturas. Pero, evidentemente, basta con añadir o sustituir las especies por las que se correspondan con otras zonas para que la base de datos se pueda trasladar a cualquier área de cualquier territorio.

Este mismo sistema es el que se ha diseñado para la cumplimentación de los campos Id_Ct (Carácter) e Id_Desc (Descripción). En este caso, el desplegable para seleccionar la opción adecuada para el campo Id_Ct proviene de la tabla CARACTER, mientras que el correspondiente al campo Id_Desc se alimenta de la tabla DESC_BOT (Figura 4). 

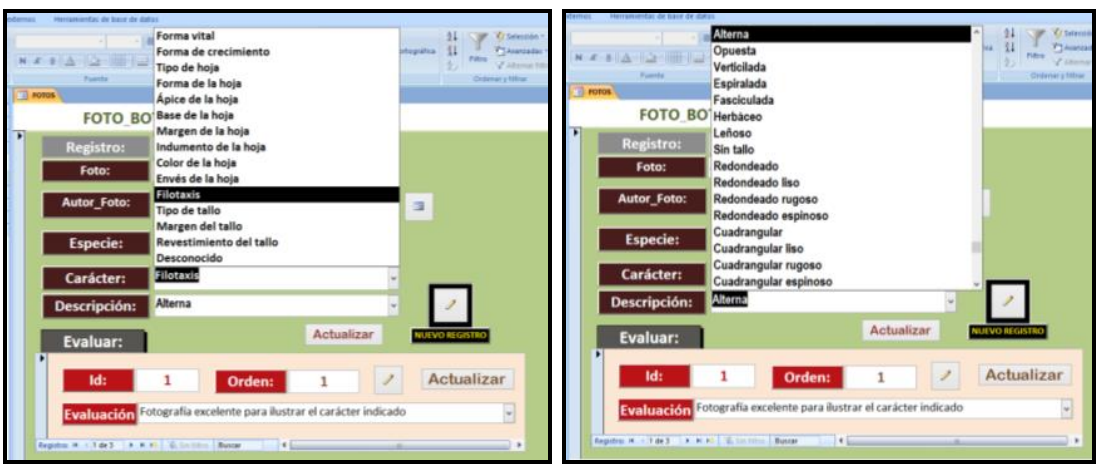

Figura 4. Recreación de las pestañas desplegables para la selección del carácter que se considera representado en la imagen (arriba) y para la descripción concreta relativa al carácter en cuestión (abajo) con los que se corresponde la fotografía.

Hasta este punto, tendríamos la descripción de la parte del formulario que debe cumplimentar el alumnado.

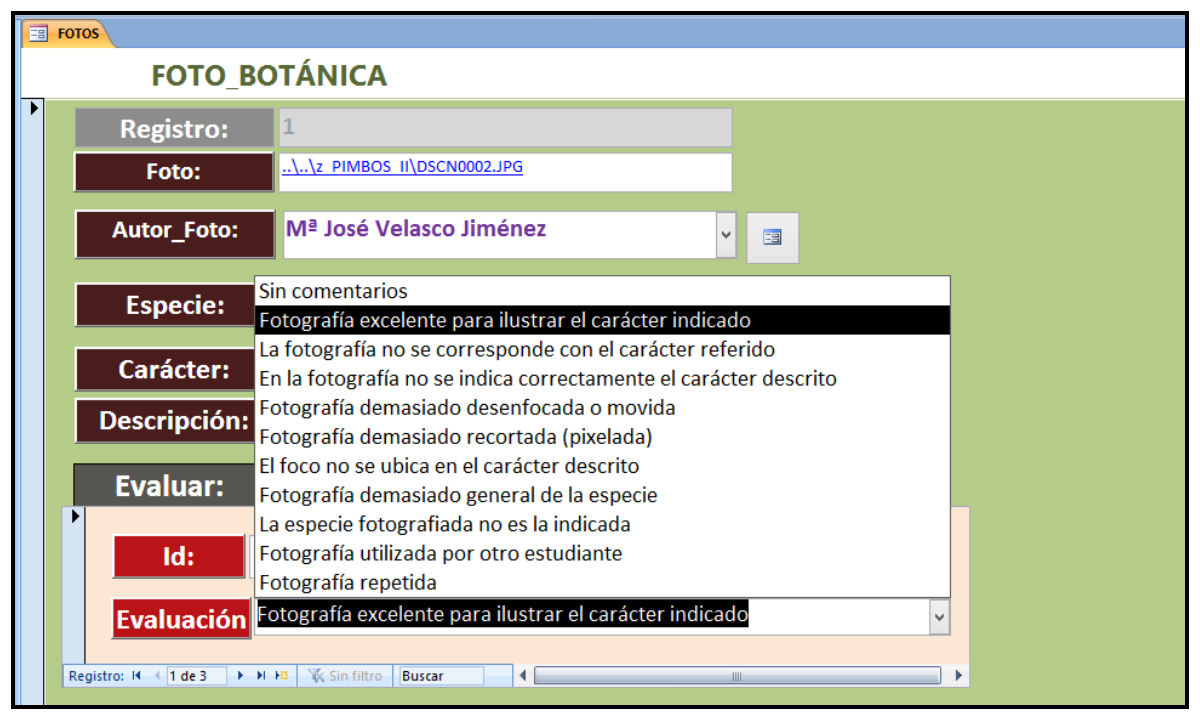

Figura 5. Recreación de la pestaña desplegable para la evaluación a cargo del profesorado.

En la parte inferior del formulario se ha diseñado un área coloreada con un tono diferente al resto en la que se incluiría la aportación del profesorado en forma de evaluación (Figura 5).

Esta parte del formulario está relacionada con la tabla EVALUA_F, que se relaciona con la tabla principal a través de la clave Id. Se implementa a través de una pestaña desplegable referida a la tabla EVALUA.

A diferencia de lo visto hasta ahora, esta parte del formulario permite la inclusión de registros múltiples. Es decir, existe la posibilidad de incluir tantos ítems de evaluación como se considere oportuno por parte del profesorado. Del mismo modo que en el resto del modelo de base de datos, no se trata de una una estructura cerrada, por lo que es posible incluir nuevos registros de evaluación en cualquier momento que se precise, a través de la tabla-diccionario "EVALUA".

\section{UTILIDAD/ANÁLISIS}

La confección de la estructura de la base de datos se ha llevado a cabo durante el curso 2016-2017, contando con el material necesario para realizar fotografías de calidad de las especies seleccionadas. Asimismo, se ha dotado de información a la propia base de datos.

La utilidad de la base de datos es doble: por un lado, se realiza un modelo cumplimentado por el profesorado que tiene un carácter demostrativo para que el alumnado pueda utilizarlo durante el tiempo dedicado al trabajo no presencial que debe realizar para asentar conocimientos y destrezas. Al tiempo, se facilita un modelo en blanco que debe ser cumplimentado por el alumnado con fotografías propias que se deben relacionar con la información adecuada en cada caso. 
Este trabajo se debe completar una vez finalizado el periodo de docencia del mismo curso, por lo que la base de datos desarrollada no se ha podido utilizar aun en las asignaturas propuestas.

Se considera factible la aplicación directa del modelo de datos que aquí se muestra en varias asignaturas impartidas desde el Departamento de Botánica, Ecología y Fisiología vegetal, que se indican en la Tabla 1. No obstante, también sería aplicable a asignaturas impartidas por otros departamentos, en grados ajenos al área de conocimiento del Departamento que suscribe este trabajo, pero con una relación evidente; como es el caso de la asignatura de Botánica Forestal y Geobotánica del Grado en Ingeniería Forestal; o en la asignatura de Botánica Agrícola, del Grado en Ingeniería Agroalimentaria y del Medio Rural.

Tabla 1. Asignaturas con uso potencial de la base de datos desarrollada.

\begin{tabular}{|l|l|}
\hline \multicolumn{1}{|c|}{ Nombre de la asignatura } & \multicolumn{1}{c|}{ Curso/Grado } \\
\hline $\begin{array}{l}\text { Principios instrumentales y metodológicos en } \\
\text { Biología de organismos y sistemas II }\end{array}$ & $1^{\circ}$ Biología \\
\hline Botánica & $2^{\circ}$ Biología \\
\hline $\begin{array}{l}\text { Bases botánicas para la gestión del medio } \\
\text { ambiente }\end{array}$ & $2^{\circ}$ Ciencias Ambientales \\
\hline Geobotánica & $4^{\circ}$ Biología \\
\hline
\end{tabular}

El hecho de que cada estudiante pueda personalizar su propia base de datos, con su propio material fotográfico $\mathrm{C}_{2}$ y que esta información pueda ser fácilmente almacenada y conservada durante mucho tiempo, hace de esta aplicación una herramienta útil para el autoaprendizaje del alumnado, que puede encontrar en ella un almacén de su propia experiencia y conocimientos sobre plantas.

Al mismo tiempo, se estima que la base de datos es una eficaz herramienta de evaluación para el profesorado, pudiendo comprobar si realmente se ha llevado a cabo un aprendizaje significativo de los conceptos y habilidades que son objeto de las asignaturas. A su vez, gracias a que la evaluación se incluye mediante items dentro de la propia base de datos, esta tendría retorno para el alumnado, con lo que se conseguiría que el proceso evaluativo incida también en el aprendizaje, llegando mucho más lejos de la simple calificación, tan necesaria como poco didáctica.

\section{CONCLUSIONES/DISCUSIÓN}

Con el modelo de base de datos que se ha presentado se ha dado un primer paso para la actualización de un método de aprendizaje clásico en el campo de estudio de la Botánica, renovándolo e incluyendo aspectos que lo mejoran de forma sustancial.

Con este proyecto se ha incorporado tecnología actual y se ha evitado la recolección de especímenes que conyeva la elaboración de herbarios tradicionales. Asimismo, se ha incidido en la verificación del aprendizaje del reconocimiento de las estructuras y otros conceptos que se desean afianzar en el alumnado y que permiten su participación activa.

\section{BIBLIOGRAFÍA}

De la Garza Vizcaya, E. L. (2004). La evaluación educativa. Revista mexicana de investigación educativa, 9(23), 807816.

Dewey, J. (1997) [1938]. Experience \& Education. Nueva York: Simon \& Schuster.

Kolb, D. A. (2001). Experietial Learning Theory Bibliography 1971-2001. Boston: McBer and Co.

López López, M. C., Hinojosa Pareja, E. F. \& Sánchez Morillo, M. D. (2014). Evaluación de la calidad de los proyectos de innovación docente universitaria, Revista de currículum y formación del profesorado, 18(3), 377-391. 